\title{
MapReduce 模型中基于直方图的数据均衡算法
}

\author{
周渭博 ${ }^{1,2,3}$, 钟勇 ${ }^{1}$, 王阳 ${ }^{1,2}$
}

$\left(\begin{array}{l}\text { 1.中国科学院成都计算机 应用研究所, 四川 成都 } 610041 ; 2 . \text { 中国科学院大学, 北京 } 100049 ; \\ \text { 3.成都崇信大数据服务有限公司, 四川成都 } 611230\end{array}\right)$

摘 要:MapReduce 模型是一种典型的分布式计算模型, 被广泛应用于大规模数据处理, 其性能很大 程度上依赖于数据分布状态。由于数据内容往往都是不均衡的, 再加上存储的随机性, 因此 MapReduce 模型在计算过程中容易出现数据倾斜的问题。针对该问题,通过改进的基于 MapReduce 的数据直方图并行构建算法, 对数据块和整个文件分别建立数据直方图, 根据数据块分布情况, 判断 每个存储节点的数据倾斜程度, 并定义了文件均衡偏差值作为数据倾斜的度量标准,进而通过数据均 衡算法来降低文件均衡偏差值。改进的基于 MapReduce 的数据直方图并行构建算法能够适应各种类 型的数据应用场景, 直方图构建过程中 Map 端向 Reduce 端只需要传输直方图统计信息, 不需要传输 文件内容, 数据传输量几乎可以忽略不计; 基于直方图的数据均衡算法采用了贪心策略, 可以获得均 衡分布最优解的一个比较好的近似解, 经过不同数据多次实验验证, 该算法与随机 block 分布算法相 比, 可以降低 $40 \%$ 左右的文件均衡偏差值, 具有更好的数据均衡效果。

\section{关 键 词: 直方图; 并行算法; 数据倾斜; 数据块; 数据均衡; 约束优化; 实验设计}

中图分类号:TP302.8

文献标志码:A

随着信息技术的发展, 人们正在以前所未有的 速度生产和消费各种各样的数据, 面对海量数据的 不断增长,传统的数据存储和计算模型无法满足需 求, 分布式系统得到了长足发展, 其中 Apache 开源 的 Hadoop ${ }^{[1]}$ 以其高可靠和低成本优势, 迅速成为业 内广泛采用的分布式解决方案。

MapReduce 模型 ${ }^{[2]}$ 是 Hadoop 的核心计算框架, 分为 Map 和 Reduce 2 个阶段: Map 阶段完成原始数 据的并行计算, 产生中间数据; Reduce 阶段读取中 间数据并计算得到最终结果。其性能取决于数据分 布状态 ${ }^{[3]}$, 数据分布比较均衡, 性能就比较高, 一旦 出现数据倾斜, 性能就会降低, 因此研究 MapReduce 模型中数据分布状态, 对提升整个分布式计算的性 能有很重要的意义。

本文通过构建数据块直方图、存储节点直方图 和文件直方图的方式来描述 MapReduce 模型中的 数据分布状态, 判断是否存在数据倾斜, 并利用贪心
文章编号: 1000-2758(2018) 03-0480-07

策略, 通过数据块交换的方法, 在不改变每个存储节 点存储量的条件下, 使数据的分布趋于均衡。

\section{1 相关研究}

\section{1 直方图}

在统计学领域, 直方图常用于描述数据的分布 状态,一般用横轴表示数据类型或数据范围, 纵轴用 一系列高度不等的纵向条纹或线段表示数据的分布 情况。直方图描述的数据分布状态可以为分布式并 行计算的效率优化奠定基础 ${ }^{[4-5]}$, 文献 [6] 中利用元 组抽样方法提出基于 MapReduce 的小波直方图构 建算法, 文献 [7] 中提出了基于 MapReduce 的 maxdiff 直方图的构建方法, 文献 [8] 中对 MapReduce 框架进行扩展, 在 $\operatorname{map}($ ) 函数之前和 reduce ( ) 函数之后分别增加了数据的采样和统计, 对基于 MapReduce 的等宽和等深直方图构建算法 
进行了改进。

上述基于 MapReduce 的直方图构建算法的核 心思想是将文件中的原始数据在 Map 阶段计算并 转换成 $<$ key, value $>$ 对的形式, 然后通过 Hash 分区 将 $<$ key, value $>$ 对传输到相应的 Reduce 节点, 最后 在 Reduce 节点上进行直方图的构建, 这必然会出现 Map 节点与 Reduce 节点间数据传输量大的问题。 尽管很多改进算法能够降低 Map 节点与 Reduce 节 点间的数据传输量, 但仍然无法从根本上解决该 问题。

\section{2 数据均衡}

MapReduce 的计算过程中可能出现 2 个方面的 性能瓶颈: Map 阶段并行计算时间受到负载重的 Map 任务制约; Reduce 阶段计算时间受到负载重的 Reduce 任务制约。文献 [9] 中分析了原因: Map 阶 段是由于原始数据分布不均衡, 导致某一个 (或某 一些) Map 任务处理的数据量远多于其他 Map 任 务; Reduce 阶段是由于数据内容本身的倾斜和 Hadoop 默认 Hash 分区方法不合理, 导致某一个 (或 某一些) Reduce 任务的数据处理量远多于其他 Reduce 任务。

Map 阶段的数据倾斜与数据内容息息相关,而 数据内容是数据自身的属性, 是无法改变的, 因此目 前大量的研究集中在 Reduce 阶段对中间数据的分 区方法改进上。例如: 文献 [10-11] 中提出基于元组 的采样策略, 文献 [12-13] 中提出基于数据块的采用 策略, 其思路都是先采样后调整分区; 文献 [14] 中 提出了一种增量式分区策略, 通过多轮递增机制实 现 Reduce 端数据平衡。

基于以上研究现状, 本文尝试通过改进的基于 MapReduce 模型的数据直方图并行构造算法完成对 Map 阶段的数据倾斜进行度量和判定的工作, 并在 此基础上通过数据均衡算法对其存在的数据倾斜问 题进行改进和优化。

\section{2 直方图构造算法}

针对 MapReduce 模型中直方图数据传输量大 的问题, 本文通过计算分组、计算频率和、传输直方 图信息等过程实现基于 MapReduce 的数据直方图 的并行构建, 从根本上解决了 Map 节点与 Reduce 节点间的数据传输量大的问题。

\section{1 相关定义}

横看成岭侧成峰, 远近高低各不同。我们在看 待事物的时候, 如果角度不同,往往会得到不同的结 果。对于数据来说, 具有同样的道理。数据本身是 有具有维度的, 同样的数据从不同的维度进行观察 往往也会得到不同的结果。在具体的数据应用场景 中, 通常是针对某一个 (或某几个) 具体的数据维度 进行数据分析, 不失一般性, 本文假设一个数据文件 datafile 中含有 mass 条记录, 存储于分布式系统中, 并据此进行数学定义:

定义 1 数据文件 datafile 中的若干条记录中 均包含某一方面的信息, 提取该信息片段,并将其定 义为数据维度 $D$, 设其对应的值域为 $R$, 且其取值可 通过计算转化为数值域 $R^{*}$ 。

例如 在分析 $\log 4 \mathrm{j}$ 产生的海量日志文件时, 其日志级别主要是 FATAL、ERROR、WARN、INFO 和 DEBUG, 我们可以将该日志文件作为 datafile, 在 其数据维度 $D$ (级别 Level) 上, 值域 $R$ 为 $\{$ FATAL, ERROR, WARN, INFO, DEBUG $\}$, 并可以进一步将 其转换成数值域 $R^{*}$ 为 $\{1,2,3,4,5\}$ 。

定义 2 数据文件 datafile 中的每条记录在值 域 $R$ (或数值域 $R^{*}$ ) 上都对应着一个值, 设为 $x$, 用 $f(x)$ 表示 datafile 中所有记录取值为 $x$ 的频率值; 另 设 $G_{i}$ 为该文件的第 $i$ 个分组, 则可以进一步定义当 $x$ $\in G_{i}$ 时, datafile 中所有记录在该分组范围内的频 率和。

$$
y=f(x), x \in G_{i}
$$

定义 3 以 $G_{i}$ 为横坐标轴, 处于该范围内的频 率和 $f(x)$ 为纵坐标轴, 建立数据文件 datafile 在数 据维度 $D$ 上对应的直方图 $H$, 其数学表达式为:

$$
H=\left\{<G_{i}, f(x)>, i \in[1, N], x \in G_{i}\right\}
$$
式中, $N$ 为分组数。

\section{2 算法描述}

\section{Step1 计算分组}

直方图横坐标轴的核心是计算文件在数据维度 $D$ 的对应值域 $R$ (或数值域 $R^{*}$ ) 的范围或类型, 并据 此进行分组, 所有并行构造直方图的 Map 节点必须 有统一的分组。根据应用条件的不同, 本文提出 3 种确定直方图横坐标范围或类型的方法:

1) 如果文件在数据维度 $D$ 的对应值域 $R$ (或数 值域 $R^{*}$ ) 的范围已知, 可以根据经验快速分组。比 如: 在分析某文件的时间维度上, 可以得到每天、每 周、每月或每年的统计分析结果, 那么其数值域可以 
相应的设置为 $1 \sim 24,1 \sim 7,1 \sim 31$ 或 $1 \sim 12$ 等。

2) 如果文件在数据维度 $D$ 的对应值域 $R$ (或数 值域 $\left.R^{*}\right)$ 的范围未知, 且相关数据维度的类型不明 确, 可以先在 Map 节点上采用相同的标准对数据块 进行并行聚类, 再根据分类情况在 Reduce 节点上统 计所有的分类。比如: 对微博数据中用户关注的领 域进行分析时, 可以先在 Map 节点并行执行领域聚 类计算, 再通过 Reduce 节点合并统计出整个文件所 有用户关注的全部领域。

3 ) 如果文件在数据维度 $D$ 的对应值域 $R$ (或数 值域 $R^{*}$ ) 的范围未知, 且数据分布不清楚, 可以在 各 Map 节点通过各自的 map task 计算每个数据块 的最大值和最小值, 然后将所有数据块对应的最大 值和最小值传输到一个 Reduce 节点, 计算出全局最 大值和全局最小值, 再据此进行分组。比如: 在分析 高中在校学生身高数据时, 就可以使用该方法来确 定直方图的横坐标轴。

\section{Step2 计算频率和}

直方图纵坐标轴的核心是计算每个分组对应的 频率和。由于数据文件被划分成若干个数据块分布 式存储, 因此在计算频率和的过程中, 本文以数据块 为单位进行计算, 可以获得每个数据块在每个横坐 标分组范围内的频率和。

\section{Step3 构造数据块直方图}

根据直方图横坐标轴的分组信息和纵坐标轴的 频率和信息, 可以在 Map 节点上并行的构造出所有 的数据块直方图。

\section{Step4 传输数据块直方图信息}

Map 节点并行构造出的数据块直方图无法直接 传输到 Reduce 节点上, 须将其转化成 $<$ key, value $>$ 对的形式,本文采用 $<G_{i}, f(x)>$ 的形式传输一个 数据块的数据直方图信息。

\section{Step5 构造文件直方图}

当所有数据块的直方图信息都传输到一个 Reduce 节点上, 该 Reduce 节点将所有数据块对应的 $<$ key, value > 对中 key 值相同的进行分组, 并依次将 对应的 value 值进行累加操作, 得到新 $<$ key, value $>$ 对,进而构造出整个数据文件的数据直方图。

基于 MapReduce 的直方图并行构造过程如图 1 所示:

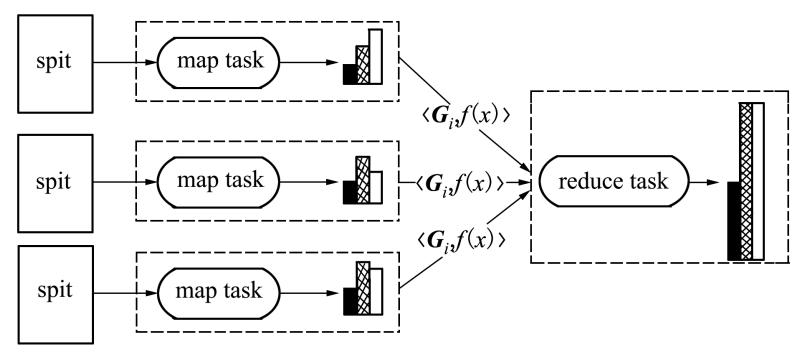

图 1 直方图并行构造过程

\section{3 基于直方图的数据均衡算法}

数据直方图可以描述出在某一数据维度 $D$ 上, 文件在分布式系统中各个存储节点上的分布情况， 根据该分布情况, 我们可以提取出相关的数学模型。

\section{1 相关定义}

定义 4 设数据文件 datafile 被划分成 $n$ 个 block, 每个 block 在建立数据直方图时划分成 $m$ 个 组,则该文件中所有 block 的数据直方图信息可以 使用以下矩阵 $\boldsymbol{A}$ 来表达:

$$
\boldsymbol{A}=\left(\begin{array}{cccc}
a_{11} & a_{12} & \cdots & a_{1 m} \\
a_{21} & a_{22} & \cdots & a_{2 m} \\
\cdots & \cdots & \cdots & \cdots \\
a_{n 1} & a_{n 2} & \cdots & a_{n m}
\end{array}\right)
$$

式中,该矩阵的第 $i$ 行表示第 $i$ 个 block 对应的数据 直方图的信息, 第 $j$ 列表示数据直方图的第 $j$ 个分 组, 数值 $a_{i j}$ 表示第 $i$ 个 block 在其第 $\mathrm{j}$ 个分组组距值 范围内的频率和信息。

由于数据文件在划分成 block 时是采用固定大 小划分的, 忽略末尾 block 差异性, 可以得到以下约 束条件:

$$
\sum_{k=1}^{k=m} a_{i k}=\sum_{k=1}^{k=m} a_{j k},(i \neq j)
$$

上述矩阵 $\boldsymbol{A}$ 及其约束条件可以看作是基于数 据块的文件直方图的一种数学表达。

如果将这 $n$ 个 block 分布存储于 $N$ 个存储节点 上 $(n \gg N)$, 在不改变每个存储节点存储容量的条 件下, 要想每个分组的频率和都尽量达到均衡分布, 问题就转变成在含有约束条件的矩阵 $\boldsymbol{A}$ 中将 $n$ 个行 向量, 分成 $N$ 份, 使得每一份中 $m$ 个分量上的值(或 累加值) 趋近于相等, 问题的本质是在不改变数据 块内容以及每个节点数据块数量的前提下, 寻找一 个数据块均衡分布的全局最优解。 


\section{定义 5 节点均衡向量 $\boldsymbol{A}_{\mathrm{avg}}$}

将 $n$ 个 block 均衡的分配到 $N$ 个节点上, 则每 个节点上的节点均衡值可以定义成一个节点均衡 向量:

$$
\begin{aligned}
& \boldsymbol{A}_{\text {avg }}=\left(\begin{array}{llll}
a v_{1} & a v_{2} & \cdots & a v_{m}
\end{array}\right)= \\
& \left(\begin{array}{llll}
\sum_{i=1}^{n} a_{i 1} & \sum_{i=1}^{n} a_{i 2} & \cdots & \sum_{i=1}^{n} a_{i m}
\end{array}\right)
\end{aligned}
$$$$
N
$$

定义 6 组合矩阵 $\boldsymbol{A}_{k}$

将矩阵 $\boldsymbol{A}$ 中的任意 $k$ 个行向量累加组合成一个 新的行向量, 构成的新矩阵定义为 $\boldsymbol{A}_{k}$, 则矩阵 $\boldsymbol{A}_{k}$ 中 必然有 $C_{n}^{k}$ 个行向量。

矩阵 $\boldsymbol{A}_{k}$ 中的行向量与均衡向量之间的相似性 的度量标准很多, 比如: 欧氏距离、曼哈顿距离、马氏 距离、夹角余弦等, 考虑到本文提出的数据均衡算法 关注的重点在于每个存储节点上相关分量的频率和 是否相等, 偏重于数值大小的比较, 因此本文选用欧 式距离作为向量间相似度的衡量标准。

设有行向量 $\boldsymbol{p}=\left(\begin{array}{llll}a_{i 1} & a_{i 2} & \cdots & a_{i m}\end{array}\right)$, 则向量 $\boldsymbol{p}$ 与均衡向量之间的欧式距离:

$d=\sqrt{\left(a_{i 1}-a v_{1}\right)^{2}+\left(a_{i 2}-a v_{2}\right)^{2}+\cdots+\left(a_{i m}-a v_{m}\right)^{2}}$

定义 7 文件均衡偏差值 $d_{\mathrm{f}}$ 将所有存储节点 中的 block 所构成的行向量与均衡向量之间的距离 累加值定义为文件均衡偏差值。

\section{2 算法描述}

$$
d_{\mathrm{f}}=d_{1}+d_{2}+\cdots+d_{N}
$$

本文提出的数据均衡算法是在不改变所有节点 已经分配 block 数量的情况下, 通过各个节点间 block 的调整和交换, 实现数据内容方面的均衡。因 此, 确定每个节点存储了多少个 block 是本算法的 前提条件。

例如 表 1 是一个被划分成 14 个 block 的文 件, 该文件被分布到 5 个节点上, 其每个节点 block 分布数量信息如表 1 所示。

\section{表 1 节点中 block 分布数量}

\begin{tabular}{cccccc}
\hline 节点 & 1 & 2 & 3 & 4 & 5 \\
\hline block 分布数量 & 3 & 3 & 3 & 3 & 2 \\
\hline
\end{tabular}

根据 block 直方图信息和 block 分布数量信息, 本文提出基于数据直方图的数据均衡算法, 具体过 程如下:
Step1 计算节点均衡向量 $\boldsymbol{A}_{\mathrm{avg}}$ ；

Step2 构造组合矩阵 $\boldsymbol{A}_{k}$;

设 block 分布信息表中 block 数最大值为 $k$, 则 需要构造组合矩阵 $\boldsymbol{A}_{k}$ 。例如: 上表所示的 block 分 布信息中, block 数最大值是 3 , 则需要构造组合矩 阵 $\boldsymbol{A}_{3}$, 且 $\boldsymbol{A}_{3}$ 有 $C_{14}^{3}=364$ 个行向量。

Step3 计算向量距离;

计算矩阵 $\boldsymbol{A}_{k}$ 中所有行向量与节点均衡向量之 间的距离;

Step4 根据距离大小分配 block。

从组合矩阵 $\boldsymbol{A}_{k}$ 中选择 $d$ 值最小的 $N$ 个互不相 关的行向量, 并将组成该行向量的若干个 block 分 配到同一个存储节点上。

算法:

Input: matrix $\boldsymbol{A}$ and block distribution quantity

Output: block distribution information

1.Aavg $[$ group $] \leftarrow \boldsymbol{A}[n][$ group $]$;

2.while $\boldsymbol{A}$ is not null and $n>k$

$/ /$ Take any $k$ rows data from matrix $\boldsymbol{A}$

$/ /$ and accumulate into one row into matrix $\boldsymbol{A}_{k}$

3. $\boldsymbol{A}_{k}[i][$ group $] \leftarrow \boldsymbol{A}[n][$ group $]$;

$/ /$ Mark the $k$ rows subscript composed of $/ / \boldsymbol{A}_{k}[i][$ group $]$ from matrix $\boldsymbol{A}$

4.Index $[i][k] \longleftarrow 1 \cdots k$;

5. $d_{i} \leftarrow \mid \boldsymbol{A}_{k}[i][$ group $]-\operatorname{Aavg}[$ group $] \mid$;

6.min $\leftarrow d_{i}$; // Search minimum value in $d_{i}$

$/ /$ Find $k$ rows data from matrix $\boldsymbol{A}$

7.A $[\operatorname{Index}[i][0]][\operatorname{group}] \cdots \boldsymbol{A}[\operatorname{Index}[i][k]]$ [group ];

$/ /$ Delete the $k$ rows data from matrix $\boldsymbol{A}$

$8 . \boldsymbol{A}[n-k][$ group $] \leftarrow \boldsymbol{A}[n][$ group $] ;$

9. $n=n-k$;

10.end while

\section{4 实验验证}

\section{1 实验设计}

为了验证算法的正确性和优越性,利用虚拟机 搭建一个由 7 个节点构成的 Hadoop 集群进行测试。 整个 Hadoop 集群由 2 个 NameNode 节点和 5 个 DataNode 节点组成, 其中每个节点均为内存 512MB, 硬盘 8GB, 操作系统为 CentOS 6.5, Hadoop 版本为 2 . 6.0 , 修改并设置 block 大小为 $256 \mathrm{kB}$, 副本数为 1 。 
Step1 采用构造数据直方图的算法构造整个 数据文件的数据直方图。将包含 1223348 个随机 数的大小为 $3.5 \mathrm{MB}$ 的数据文件 datafile. txt 存储到 实验中搭建的 Hadoop 集群中, 设置分组数为 5 , 构 建数据块数据直方图和整个文件数据直方图, 分析 数据倾斜状态, 并对比直方图构造算法的数据传 输量。

Step2 根据整个文件的数据直方图和每个数 据块 (block) 的数据直方图的相关信息, 采用随机分 配和基于数据直方图的数据均衡算法进行分配 2 种 方案, 对比其文件均衡偏差值, 并根据节点数据直方 图分析其数据倾斜状态。

\section{2 实验结果及分析}

\subsection{1 直方图分析}

由于实验集群中 block 大小被设置为 $256 \mathrm{kB}$, 因此实验中的数据文件被划分成 14 个 block, 每个 block 对应的分组和每组频率和的统计信息如表 2 所示:

表 2 block 统计信息

\begin{tabular}{cccccc}
\hline block 序号 & $0 \sim 20$ & $21 \sim 40$ & $41 \sim 60$ & $61 \sim 80$ & $81 \sim 100$ \\
\hline 1 & 17618 & 17495 & 17363 & 17404 & 17502 \\
2 & 8662 & 17453 & 26488 & 21986 & 12793 \\
3 & 2647 & 26301 & 38354 & 9512 & 10568 \\
4 & 13110 & 21893 & 26485 & 21551 & 4343 \\
5 & 4378 & 12943 & 8982 & 17396 & 43683 \\
6 & 14010 & 13909 & 24430 & 24489 & 10544 \\
7 & 10433 & 13915 & 24504 & 31597 & 6933 \\
8 & 10513 & 26103 & 21041 & 27954 & 1771 \\
9 & 7089 & 17320 & 33126 & 19285 & 10562 \\
10 & 13242 & 21782 & 17532 & 24355 & 10471 \\
11 & 11303 & 23628 & 13155 & 20049 & 19247 \\
12 & 13993 & 14081 & 29469 & 19259 & 10580 \\
13 & 24460 & 19253 & 15812 & 26983 & 874 \\
14 & 26117 & 8524 & 26319 & 8789 & 17633 \\
\hline
\end{tabular}

通过表 2 可以直接构建出每个 block 的数据直 方图,如图 2 所示:

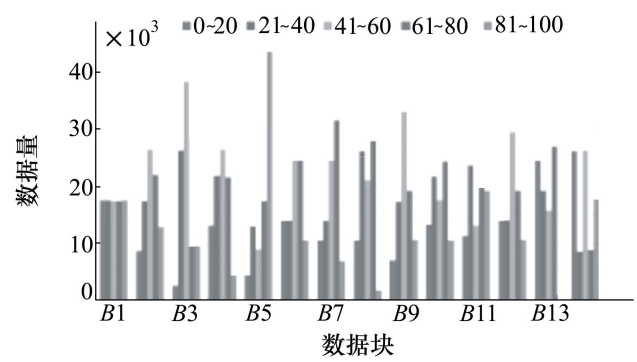

所有 block 数据直方图的信息在 Reduce 节点合 并统计后, 得到整个文件的数据直方图, 如图 3 所示:

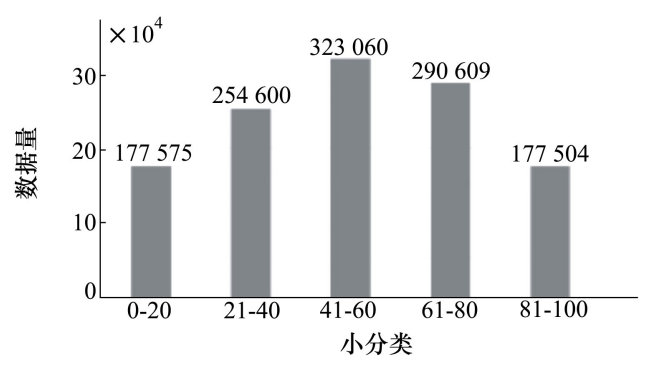

图 3 file 数据直方图

通过 block 的数据直方图 (见图 2) 可以看出: 多 个 block 之间的数据分布差异明显; 通过整个文件 的数据直方图 (见图 3 ) 可以看出: 整个文件的数据 内容本身分布不均衡,第 3 个分组的数据量明显偏 多,第 1 个分组和第 5 个分组的数据量明显偏少。

算法数据传输量分析: 如果采用传统的直方图 构建算法,需要将 3.5 MB 的数据在 Map 端处理成 120 多万个 $<$ key, value $>$ 对, 然后传输到 Reduce 端, 通过 $<$ key, value $>$ 对合并来完成文件直方图的构建; 而采用本文提出的改进算法只需要传输 14 个数据 块共 70 个 $<\mathrm{key}$, value $>$ 对的统计信息, 就可以在 Reduce 端快速建立整个文件的直方图，而且还可以同 时完成数据块直方图的构建和该文件在某一存储节 点上数据直方图的构建。

\subsection{2 数据均衡分析}

将 14 个 block 分布到 5 个存储节点上, 假设 block 分布情况为: 4 个三 block 节点, 1 个双 block 节点,对比 2 种 block 分布算法。

方案 1 随机分布算法

表 3 随机算法 block 分布状态

\begin{tabular}{cccccc}
\hline block & $0 \sim 20$ & $21 \sim 40$ & $41 \sim 60$ & $61 \sim 80$ & $81 \sim 100$ \\
\hline number & 3 & 3 & 3 & 3 & 2 \\
& block1 & block4 & block7 & block10 & block13 \\
distribution block2 & block5 & block8 & block11 & block14 \\
& block3 & block6 & block9 & block12 & \\
\hline
\end{tabular}

文件均衡偏差: $d_{\mathrm{f}}=137467.08$

方案 2 数据均衡算法

图 2 block 数据直方图 


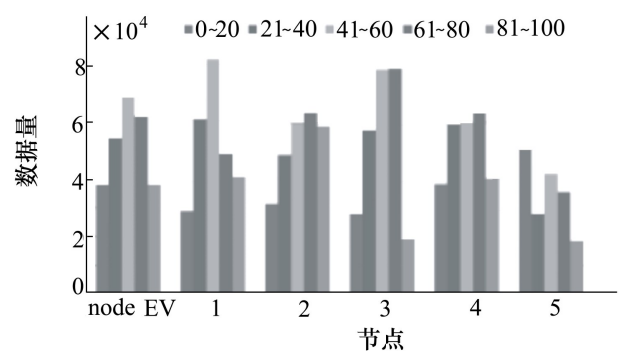

图 4 随机算法节点直方图

表 4 均衡算法 block 分布状态

\begin{tabular}{cccccc}
\hline block & $0 \sim 20$ & $21 \sim 40$ & $41 \sim 60$ & $61 \sim 80$ & $81 \sim 100$ \\
\hline number & 3 & 3 & 3 & 3 & 2 \\
& block1 & block6 & block2 & block3 & block4 \\
distribution block9 & block11 & block8 & block5 & block7 \\
block10 & block12 & block14 & block13 & \\
\hline
\end{tabular}

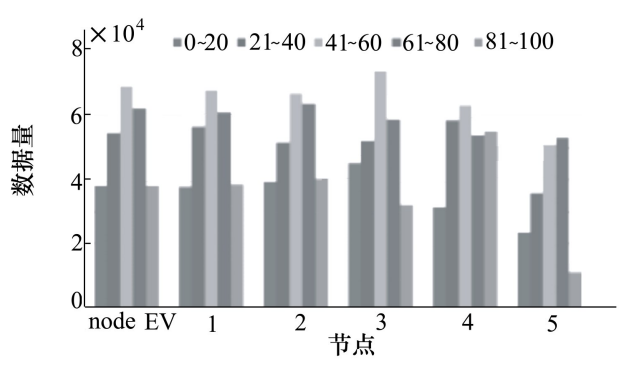

图 5 均衡算法节点直方图

文件均衡偏差: $d_{\mathrm{f}}=81290.32$

图 4 和图 5 中的 nodeEV 表示节点期望值, 通过
nodeEV 与 node1 node5 的对比可以看出: 采用随机 block 分布算法, node1 node5 节点之间以及节点与 nodeEV 之间直方图差异比较明显; 而采用数据均衡 算法, node1 node5 节点之间直方图差异比较小, 而 且基本上与 nodeEV 的直方图分布很接近, 因此采 用数据均衡算法比采用随机 block 分布算法具有更 好的数据均衡效果。

经过不同数据多次实验验证,采用数据均衡算 法比采用随机 block 分布算法在文件均衡偏差值上 可以有较大幅度的提高 (本文实验结果提高了 $40.87 \%$ )。

\section{5 结 论}

本文针对 MapReduce 并行计算框架中出现的 中间数据的数据倾斜问题, 提出了一种基于直方图 的数据均衡算法, 该算法通过构建数据块直方图的 形式来分析数据倾斜问题, 并定义文件均衡偏差值 对数据倾斜程度进行度量, 进而通过交换节点中存 储的数据块的方式, 在保证所有节点存储容量不变 的条件下,降低文件均衡偏差值。经过不同数据多 次实验验证, 该算法比随机 block 分布算法具有更 好的均衡效果。

同时也要指出, 本文提出基于直方图的数据均 衡算法在计算过程中采用了贪心策略,该策略并不 保证能够找到数据均衡分布的全局最优解, 但是可 以找到一个全局最优解的很好的近似解, 因此在工 程上具有极大的应用价值。

\section{参考文献:}

[1] 陈吉荣,乐嘉锦. 基于 Hadoop 生态系统的大数据解决方案综述 $[\mathrm{J}]$. 计算机工程与科学, 2013, 35(10): 25-35

Chen Jirong, Le Jiajin. Reviewing the Big Data Solution Based on Hadoop Ecosystem[J]. Computer Engineering and Science, 2013, 35(10): 25-35 (in Chinese)

[2] 李建江, 崔健, 王聘, 等. MapReduce 并行编程模型研究综述 $[\mathrm{J}]$. 电子学报, 2011, 39(11):2635-2642

Li Jianjiang, Cui Jian, Wang Dan, et al. Survey of MapReduce Parallel Programming Model [J]. Acta Electronica Sinica, $2011,39(11): 2635-2642$ (in Chinese)

[3] 王刚, 李盛恩. MapReduce 中数据倾斜解决方法的研究 [J]. 计算机技术与发展, 2016, 26(9):201-204

Wang Gang, Li Sheng' en. Research on Handling Data Skew in MapReduce[J]. Computer Technology and Development, 2016, 26(9):201-204 (in Chinese)

[4] Blanas S, Patel J M, Ercegovac V, et al. A Comparison of Join Algorithms for Log Processing in MapReduce[ C] //Proceedings of the 2010 ACM SIGMOD International Conference on Management of Data, New York, 2010: 975-986

[5] Zhang Chi, Li Feifei, Jestes J. Efficient Parallel KNN Joins for Large Data in MapReduce[C] //Proceedings of the 15th International Conference on Extending Database Technology, New York, 2012:38-49 
[6] Jestes J, Yi Ke, Li Feifei. Building Wavelet Histograms on Large Data in MapReduce[ C] // Proceedings of the Vldb Endowment, 2011: 109-120

[7] Shi Yingjie, Meng Xiaofeng, Wang Fusheng, et al. HEDC++: an Extended Histogram Estimator for Data in the Cloud[J]. Journal of Computer Science and Technology, 2013, 28(6) :973-988

[8] Tang Mingwang. Efficient and Scalable Monitoring and Summarization of Large Probabilistic Data[C]// Proceedings of the 2013 SIGMOD/PODS Ph D Symposium, New York, 2013:61-66

[9] Kwon Y, Balazinska M, Howe B, et al. A Study of Skew in MapReduce Applications[C] // Open Cirrus Summit, Moscow, Russia, 2011

[10] Gufler B, Augsten N, Reiser A, et al. Load Balancing in MapReduce Based on Scalable Cardinality Estimates[C] //Proceedings of the 2012 IEEE 28th International Conference on Data Engineering( ICDE), Washington, USA, 2012:522-533

[11] Gufler B, Augsten N, Reiser A, et al. Handing Data Skew in MapReduce[C]// Proceedings of the 1st International Conference on Cloud Computing and Services Science, Noordwijkerhout, Netherlands, 2011, 146:574-583

[12] Kolb L, Thor A, Tahm E. Block-Based Load Balancing for Entity Resolution with MapReduce[ C] //Proceeding of the 20th ACM International Conference on Information and Knowledge Management Glasgow, UK, 2011:2397-2400

[13] Kolb L, Thor A, Tahm E. Load Balancing for MapReduce-Based Entity Resolution[C]//Proceedings of the 2012 IEEE 28 th International Conference on Data Engineering, Washington, USA, 2012:618-629

[14] 王卓, 陈群, 李战怀, 等. 基于增量式分区策略的 MapReduce 数据均衡方法 $[\mathrm{J}]$. 计算机学报, 2016(1):19-35 Wang Zhuo, Chen Qun, Li Zhanhuai, et al. An Incremental Partitioning Strategy for Data Balance on MapReduce[J]. Chinese Journal of Computers, 2016(1):19-35 (in Chinese)

\title{
Data Balance Algorithm Based on Histogram in MapReduce
}

\section{Zhou Weibo ${ }^{1,2,3}$, Zhong Yong $^{1}$, Wang Yang ${ }^{1,2}$}

\author{
1.Chengdu Institute of Computer applications, Chinese Academy of Sciences, Chengdu 610041, China; \\ 2. University of Chinese Academy of Sciences, Beijing 100049, China; \\ 3. Chengdu ChongXin Big Data Services Co., Ltd. Chengdu 611230, China
}

Abstract: MapReduce model is a typical distributed computing model, which is widely used in large-scale data processing, and its performance depends largely on the data distribution status. As the data content is often unbalanced, coupled with the storage of randomness, so MapReduce model prone to data skew problem in the calculation process. In order to solve this problem, this paper establishes a data histogram for the data block and the whole file through the improved parallel histogram parallelization algorithm based on MapReduce. According to the data block distribution, we can judge the data skew degree of each storage nodes and define the file equilibrium deviation value as the measure of data skew, and then the data balance algorithm is used to reduce the file equilibrium deviation value. The improved MapReduce-based data histogram parallel construction algorithm can adapt to various types of data application scenarios. In the process of building the histogram, the Map side only needs to transmit histogram statistics to the Reduce side without transmitting the contents of the file. The data transfer can be almost negligible. The data balance algorithm based on histogram employs greedy strategy, which can obtain a better approximate solution of the optimal solution of equilibrium distribution. After several experiments, compared with the random block distribution algorithm, the improved algorithm reduce about $40 \%$ of the file balance deviation value and achieves a better data balance performance.

Keywords : histogram ; parallel algorithm ; data skew ; block ; data balance ; constrained optimization ; design of experiments 